Phase transition in the 3D massive Gross-Neveu model

This article has been downloaded from IOPscience. Please scroll down to see the full text article.

2010 EPL 9211001

(http://iopscience.iop.org/0295-5075/92/1/11001)

View the table of contents for this issue, or go to the journal homepage for more

Download details:

IP Address: 200.128.60.106

The article was downloaded on 02/08/2013 at 13:36

Please note that terms and conditions apply. 


\title{
Phase transition in the 3D massive Gross-Neveu model
}

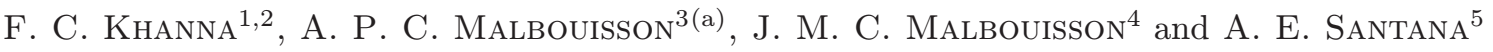 \\ ${ }^{1}$ Department of Physics, Theoretical Physics Institute, University of Alberta - Edmonton, Alberta, T6G 2J1, Canada \\ 2 TRIUMF - 4004, Westbrook mall, Vancouver, British Columbia V6T 2A3, Canada \\ ${ }^{3}$ Centro Brasileiro de Pesquisas Físicas, MCT - 22290-180, Rio de Janeiro, RJ, Brazil \\ ${ }^{4}$ Instituto de Física, Universidade Federal da Bahia - 40210-340, Salvador, BA, Brazil \\ ${ }^{5}$ Instituto de Física, International Center for Condensed Matter Physics, Universidade de Brasilia \\ 70910-900, Brasilia, DF, Brazil
}

received 24 August 2010; accepted 22 September 2010

published online 29 October 2010

PACS 11.10.Kk - Field theories in dimensions other than four

PACS 11.30.Qc - Spontaneous and radiative symmetry breaking

PACS 11.10.Wx - Finite-temperature field theory

\begin{abstract}
We consider the 3-dimensional massive Gross-Neveu model at finite temperature as an effective theory for strong interactions. Using the Matsubara imaginary-time formalism, we derive a closed form for the renormalized $T$-dependent four-point function. This gives a singularity, suggesting a phase transition. Considering the free energy we obtain the $T$-dependent mass, which goes to zero for some temperature. These results lead us to the conclusion that there is a secondorder phase transition.
\end{abstract}

Introduction. - The Gross-Neveu (GN) model [1], proposed as an effective model for quantum chromodynamics (QCD), has been analyzed extensively in recent years. In several cases, these studies consider the temperature effect, associated with fermion systems, in both particle and condensed-matter physics [2-18]. The motivations and the nature of such analysis are multiple, including investigations of the continuous and discrete chiral symmetry $[6,7]$. The version with $N$ massless fermions in $(2+1)$ dimensions, for instance, presents chirality breaking in perturbative analysis, and its restoration at finite temperature [14]. This provides insight into the intricate structure of the hadronic matter, such as for the quark confinement/deconfinement transition [5,15-17]. Due to such characteristics, the GN model is taken as a prototype for analysis of phase transitions in quantum field theory for fermions. In this sense, the search for analytical results is another aspect that attracts interest, in general [18], but in particular for $N=1$, where lattice calculations are hard to carry out. In this note, we address the $(2+1)$-dimensional GN model at finite temperature, but with non-zero mass and one type of fermion.

The massive GN model in $(2+1)$ dimensions was considered in [19], even though this version for dimensions greater than two is perturbatively non-renormalizable.

\footnotetext{
(a) E-mail: adolfo@cbpf.br
}

This is justified by considering that, perturbative renormalizability is not an absolute criterion for an effective theory to be physically consistent. This fact is known and has been used for long [20-25]. Here we will consider the $(2+1)$-dimensional massive GN model at finite temperature with an arbitrary zero-temperature coupling constant, as an effective theory for QCD. We will take both the thermal mass and the thermal coupling constant at one-loop order. This approximation will be justified by a non-perturbative analysis of the four-point function, by summing the chains of loop diagrams. This provides a non-perturbative relationship between the four-point function and temperature. Such a relation leads to a singularity, suggesting a possible phase transition [26]. In order to get the nature of the phase transition, an analysis of the free energy is carried out. It gives rise to a $T$-dependent mass that goes to zero for some values of temperature, suggesting a second-order phase transition. An additional argument in favour of a second-order phase transition comes from an analysis of the beta-function. The argument relies on the fact that this analysis gives the result that there is a non-trivial infrared stable fixed point.

Such results bring up an interesting question: can such a study of an effective model yield any information about a phase transition in physically relevant situations for QCD? In order to get an answer to this question, we rely on 
a procedure that follows methods first developed for the BCS model in superconductivity [26].

The paper is organized in the following way. In the second section, the model is defined. In the third section, the thermal correction of the mass at the one-loop level is calculated. In the fourth section, using the temperaturedependent coupling constant, an expression for the thermal mass is derived. In the fifth section, starting with non-perturbative results for the four-point function, the analysis of the critical region is discussed. The concluding remarks are presented in the last section.

The model. - In a $D$-dimensional Euclidean manifold, $\mathbb{R}^{D}$, we consider the Hamiltonian for the massive GN model,

$$
\begin{aligned}
H= & \int \mathrm{d}^{D} x\left\{\psi^{\dagger}(x)\left(\gamma^{j}\left(i \partial_{j}\right)\right) \psi(x)-m_{0} \psi^{\dagger}(x) \psi(x)\right. \\
& \left.+\frac{\lambda_{0}}{2}\left[\psi^{\dagger}(x) \psi(x)\right]^{2}\right\},
\end{aligned}
$$

where $m_{0}$ and $\lambda_{0}$ are respectively the physical zerotemperature mass and coupling constant, $x \in \mathbb{R}^{D}$ and the $\gamma$-matrices are elements of the Clifford algebra (natural units $\hbar=c=k_{B}=1$ are used). This Hamiltonian is obtained using conventions for Euclidean field theories in [27].

From eq. (1), introducing the thermally corrected mass,

$$
m(T)=m_{0}+\Sigma(T),
$$

we have a free energy density of the Ginzburg-Landau type [28],

$$
\mathcal{F}=a+b(T) \phi^{2}(x)+c \phi^{4}(x),
$$

where $b(T)=-m(T)$ and $c=\lambda_{0} / 2$. The minus sign for the mass in eq. (3) implies that, in the disordered phase we have $m(T)<0$ and for the ordered phase $m(T)>0$, consistently. The second-order phase transition occurs at the temperature where $m(T)$ changes sign from negative to positive, characterizing a spontaneous symmetry breaking. In this formalism, the quantity $\phi(x)=\sqrt{\psi^{\dagger}(x) \psi(x)}$ plays the role of the order parameter for the transition.

Thermal self-energy. - We evaluate the thermal selfenergy, $\Sigma(T)$, by using the Matsubara imaginary-time formalism. The Cartesian coordinates are specified by $x=\left(x^{0}=\tau, \mathbf{x}\right)$, where $\mathbf{x}$ is a $(D-1)$-dimensional vector. The conjugate momentum of $x$ is denoted by $k=\left(k_{0}, \mathbf{k}\right)$, $\mathbf{k}$ being a $(D-1)$-dimensional vector in momentum space. The KMS (Kubo, Martin, Schwinger) condition implies that the Feynman rules are modified by the well-known Matsubara prescription, [29]

$$
\int \frac{\mathrm{d} k_{0}}{2 \pi} \rightarrow \frac{1}{\beta} \sum_{n=-\infty}^{+\infty}, \quad k_{0} \rightarrow \frac{2 \pi\left(n+\frac{1}{2}\right)}{\beta} \equiv \omega_{n}
$$

where $\omega_{n}$ are Matsubara frequencies and $\beta=1 / T, T$ being the temperature.
At the one-loop level the finite-temperature self-energy is given by

$$
\Sigma(D, T, s)=\lambda_{0} \frac{m_{0}}{\beta} \sum_{n=-\infty}^{\infty} \int \frac{\mathrm{d}^{D-1} k}{(2 \pi)^{D-1}} \frac{1}{\left(\mathbf{k}^{2}+\omega_{n}^{2}+m_{0}^{2}\right)^{s}} .
$$

In order to use the dimensional regularization procedure, we introduce dimensionless quantities,

$$
q_{j}=k_{j} / 2 \pi m_{0}, \quad j=1,2, \ldots, D-1 \text { and } a=\left(m_{0} \beta\right)^{-2} .
$$

For the present case, we have $D=3$ and $s=1$. After dimensional regularization [27], we obtain

$$
\Sigma(D, T, s)=\frac{m_{0} \lambda_{0} \Gamma(\nu)}{(4 \pi)^{(D-1) / 2} \Gamma(s) \beta} \sum_{n=-\infty}^{\infty}\left(\omega_{n}^{2}+m_{0}^{2}\right)^{-\nu},
$$

where $\nu=s-(D-1) / 2$. The sum in eq. (6) is cast in the general form

$$
\sum_{n=-\infty}^{+\infty}\left[a\left(n+\frac{1}{2}\right)^{2}+c^{2}\right]^{-\nu}=4^{\nu} Z_{1}^{4 c^{2}}(\nu, a)-Z_{1}^{c^{2}}(\nu, a)
$$

where

$$
Z_{1}^{b^{2}}(\nu, a)=\sum_{n=-\infty}^{+\infty}\left[a n^{2}+b^{2}\right]^{-\nu}
$$

is the generalized Epstein zeta-function [30] defined for $\operatorname{Re}(\nu)>1 / 2$. We then analytically continue $Z_{1}^{b^{2}}(\nu, a)$ to the whole complex $\nu$-plane [31-34]:

$$
\begin{aligned}
Z_{1}^{b^{2}}(\nu, a)= & \frac{\sqrt{\pi}}{\sqrt{a} \Gamma(\nu)}\left[\frac{\Gamma(\nu-1 / 2)}{b^{2 \nu-1}}\right. \\
& \left.+4 \sum_{n=1}^{\infty}\left(\frac{\pi n}{b \sqrt{a}}\right)^{\nu-\frac{1}{2}} K_{\nu-\frac{1}{2}}\left(\frac{2 \pi b n}{\sqrt{a}}\right)\right],
\end{aligned}
$$

where $K_{\alpha}(x)$ is the Bessel function of the third kind. As a consequence, $\Sigma(D, T, s)$ reads

$$
\Sigma(D, T, s)=\widetilde{\Sigma}(D, s) \frac{m_{0}^{1-2 \nu}}{\beta(2 \pi)^{2 \nu}}\left[4^{\nu} Z_{1}^{4 c^{2}}(\nu, a)-Z_{1}^{c^{2}}(\nu, a)\right]
$$

where $c=1 / 2 \pi$. Using eq. (9) in eq. (10), we obtain $\Sigma(D, T, s)$ as

$$
\Sigma(D, T, s)=F_{0}(D, s)+F_{1}(D, a, s) .
$$

Here $F_{0}(D, s)$ is a term independent of $a$, arising from the first term in brackets in eq. (9), while $F_{1}(D, a, s)$ is the term arising from the sum in the second term. We find that, for $s=1$ and even dimensions $D \geqslant 2$, $F_{0}(D, s)$ is divergent due to the pole of the $\Gamma$-function. Accordingly, this term is subtracted to get the physical $a$-dependent function $\Sigma_{R}(D, a)$. The mass counterterm is a pole appearing at the physical value $s=1$. 
The $a$-dependent correction to the mass is proportional to the regular part of the analytical extension of the Epstein zeta-function in the neighborhood of the pole at $s=1$. For uniformity, the term $F_{0}(D)$ is also subtracted in the case of odd dimensions $D$, where no poles of $\Gamma$-functions are present. Therefore, using the modified Bessel function for $D=3, s=1$ and $a=\left(m_{0} / T\right)^{-2}$, i.e. $K_{ \pm 1 / 2}(z)=\sqrt{\pi} e^{-z} / \sqrt{2 z}$, the physical thermal self-energy for $D=3$ is given by

$$
\Sigma_{R}(3, T, 1)=\frac{1}{2 \pi} \lambda_{0} m_{0}^{2} f(T),
$$

where

$$
f(T)=-\frac{T}{m_{0}} \ln \left(1+e^{-m_{0} / T}\right)
$$

The behavior of $f(T)$ is the following: for $T \rightarrow 0, f(T) \rightarrow 0$; for $T \rightarrow \infty, f(T) \rightarrow-\infty$, with $f(T)<0$ for all values of $T>0$. Using eq. (2), the temperature-dependent mass is

$$
m(T)=m_{0}+\frac{1}{2 \pi} \lambda_{0} m_{0}^{2} f(T) .
$$

The condition for a phase transition, $m(T)=0$, provides a critical temperature for each fixed value of the zerotemperature coupling constant, $\lambda_{0}$. This result indicates a second-order phase transition. However, in order to establish this result on a firmer ground, we consider the $T$-dependent correction to the coupling constant $\lambda_{0}$.

$\boldsymbol{T}$-dependent coupling constant. - Initially, we consider the four-point function with null external momenta, which defines the temperature-dependent coupling constant. It is given up to one-loop by

$$
\Gamma_{D}^{(4)}\left(\lambda_{0}, \beta\right) \simeq \lambda_{0}\left[1+\lambda_{0} \Pi(D, \beta)\right]
$$

where $\Pi(D, \beta)$ is the $\beta$-dependent one-loop polarization diagram given by

$$
\Pi(D, \beta)=\frac{1}{\beta} \sum_{n=-\infty}^{\infty} \int \frac{\mathrm{d}^{D-1} k}{(2 \pi)^{D-1}} \frac{m_{0}^{2}-\left(\mathbf{k}^{2}+\omega_{n}^{2}\right)}{\left(\mathbf{k}^{2}+\omega_{n}^{2}+m_{0}^{2}\right)^{2}} .
$$

Using the dimensionless quantities introduced in eq. (5), $\Pi(D, \beta)$ is given as

$$
\begin{aligned}
\Pi(D, a)= & \left.\Pi(D, a, s)\right|_{s=2}=\frac{m^{D-2(s-1)}}{(2 \pi)^{2}} \sqrt{a} \\
& \times\left[\frac{1}{2 \pi^{2}} U_{D}(s ; a)-U_{D}(s-1 ; a)\right]_{s=2},
\end{aligned}
$$

where

$$
\begin{aligned}
U_{D}(\mu ; a)= & \pi^{\frac{(D-1)}{2}} \frac{\Gamma(\mu-(D-1) / 2)}{\Gamma(\mu)} \\
& \times \sum_{n=-\infty}^{\infty}\left[a\left(n+\frac{1}{2}\right)^{2}+(2 \pi)^{-2}\right]^{(D-1) / 2)-\mu}
\end{aligned}
$$

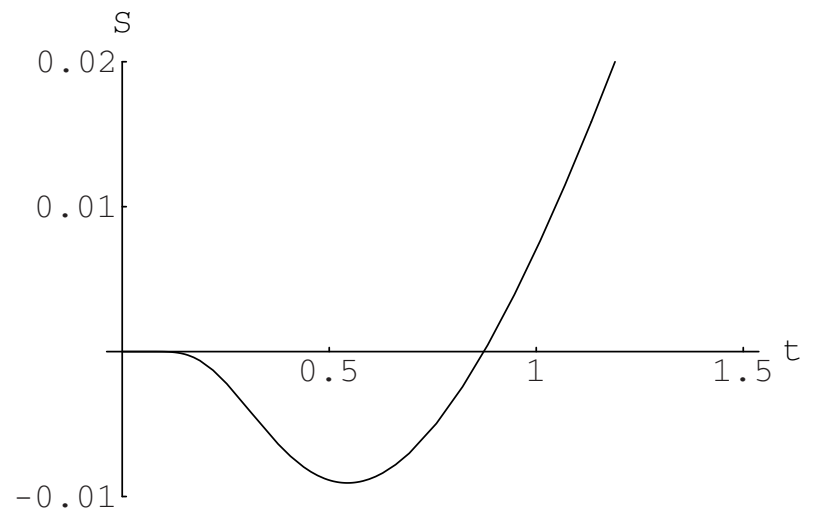

Fig. 1: Polarization (in units of $\left.m_{0}\right), S=\Pi_{R}(3, T) / m_{0}$, as a function of $T$ (in units of $m_{0}$, i.e. $t=T / m_{0}$ ).

The function $U_{D}(\mu, a)$ is extended to the whole complex $\mu$-plane, resulting in

$$
U_{D}(\mu, a)=\frac{h(\mu, D)}{\sqrt{a}}\left[\Gamma\left(\mu-\frac{D}{2}\right)+4 W(\mu, a, D)\right],
$$

where $h(\mu, D)=\pi^{2 \mu-D / 2} /\left(2^{D-2 \mu} \Gamma(\mu)\right)$ and

$$
\begin{aligned}
W(\mu, a, D)= & 2 \sum_{n=1}^{\infty}\left(\frac{\sqrt{a}}{n}\right)^{\frac{D}{2}-\mu} K_{\frac{D}{2}-\mu}\left(\frac{2 n}{\sqrt{a}}\right) \\
& -\sum_{n=1}^{\infty}\left(\frac{2 \sqrt{a}}{n}\right)^{\frac{D}{2}-\mu} K_{\frac{D}{2}-\mu}\left(\frac{n}{\sqrt{a}}\right) .
\end{aligned}
$$

Using these results in eq. (17), we obtain $\Pi(D, a, s)$ as

$$
\Pi(D, a, s)=H(D, s)+G(D, a, s)
$$

where $H(D, s)$ is a term independent of $a$, arising from the first term in brackets in eq. (19), while the term $G(D, a, s)$ arises from the the $W$-functions. As in the case of the thermal self-energy, the physical thermal polarization is obtained by subtracting the term $H(D, s)$ in eq. (21) and is given by

$$
\Pi_{R}(D, a)=\frac{m_{0}^{D-2}}{2^{D-2} \pi^{D / 2}}[2 \pi W(2, a, D)-W(1, a, D)] .
$$

Taking $D=3$ and $a=\left(m_{0} / T\right)^{-2}$, we obtain

$$
\Pi_{R}(3, T)=\frac{m_{0}}{2 \pi}\left[\frac{T}{m_{0}} \ln \left(1+e^{-m_{0} / T}\right)-\frac{1}{1+e^{m_{0} / T}}\right] .
$$

Substituting this expression into eq. (15), we obtain the temperature-dependent coupling constant,

$$
g\left(T ; \lambda_{0}\right) \equiv \Gamma_{3 R}^{(4)}\left(\lambda_{0}, T\right) \simeq \lambda_{0}\left[1+\lambda_{0} \Pi_{R}(3, T)\right]
$$

The physical polarization $\Pi_{R}(3, T)$ is shown in fig. 1 . We find from this plot and from eq. (24) that $g(T=$ $\left.0 ; \lambda_{0}\right)=\lambda_{0}$; this just reflects the fact that $\lambda_{0}$ is the physical coupling constant at $T=0$. 
The temperature-dependent coupling constant, $g\left(T ; \lambda_{0}\right)$, is introduced in place of $\lambda_{0}$ in eq. (14). Then we get a $T$-dependent mass,

$$
m(T)=m_{0}\left[1+\frac{1}{2 \pi} g\left(T ; \lambda_{0}\right) m_{0} f(T)\right] .
$$

We have then all the elements to analyze the phase transition.

The phase transition. - Let us first remind an essential feature of a situation in condensed-matter physics, theoretically close to the one we examine in this article; namely, the BCS field-theoretical approach to the superconducting phase transition [26]. In this case it is shown that, at criticality, the leading contribution to the fourpoint function with zero external momenta is given by the sum of all chains of one-loop diagrams. This nonperturbative calculation leads to an expression of the form

$$
\Gamma_{3 R}^{(4)}\left(\lambda_{0}, T\right)=\frac{\lambda_{0}}{\left[1-\lambda_{0} \Pi_{R}(3, T)\right]} .
$$

In our case the first two terms of the expansion in powers of $\lambda_{0}$ of such a function are given in eq. (24). The existence of a singularity of the four-point function in eq. (26) indicates a phase transition, as explained in [26]; in other words the phase transition is characterized by the poles of $\Gamma_{3 R}^{(4)}\left(\lambda_{0}, T_{c}\right)$, i.e.,

$$
\lambda_{0} \Pi_{R}(3, T)=1
$$

In our case this singularity is found from an analysis of fig. 1. The nature of the transition is obtained by a study of the free energy, eq. (3).

In the present article we have a situation analogous to the BCS theory, and we have done the study of the free energy for a second-order phase transition starting from eq. (3). Then the critical region is defined by taking $m\left(T_{c}\right)=0$, in eq. (25), which leads to

$$
g\left(T_{c} ; \lambda_{0}\right)=-\frac{2 \pi}{m_{0} f\left(T_{c}\right)} .
$$

This provides $T_{c}$ as a function of $\lambda_{0}$, giving a curve plotted in fig. 2. On the curve we have $m\left(T_{c}\right)=0$; below the curve we have $m(T)>0$ and above the curve $m(T)<0$. We find that the sign for the thermal mass corresponds to the expected behavior of a phase transition from a disordered to an ordered phase, as temperature is lowered.

Moreover, eq. (27) gives rise to a function $t=t(\lambda)$ (where again $\lambda=\lambda_{0} m_{0} / 2 \pi$ and $t=T / m_{0}$ ), with a behavior that is similar to that presented in fig. 2. Indeed, from fig. 1 , we obtain that $t \rightarrow 0.87$ for $\lambda_{0} \rightarrow \infty$. On the other hand, $t \rightarrow \infty$ for $\lambda \rightarrow 0$. This non-perturbative result is then compatible with the analysis performed in the previous sections, of the mass as a function of the temperature, taking the thermal corrections to both the mass and the coupling constant at one-loop level. Then we conclude

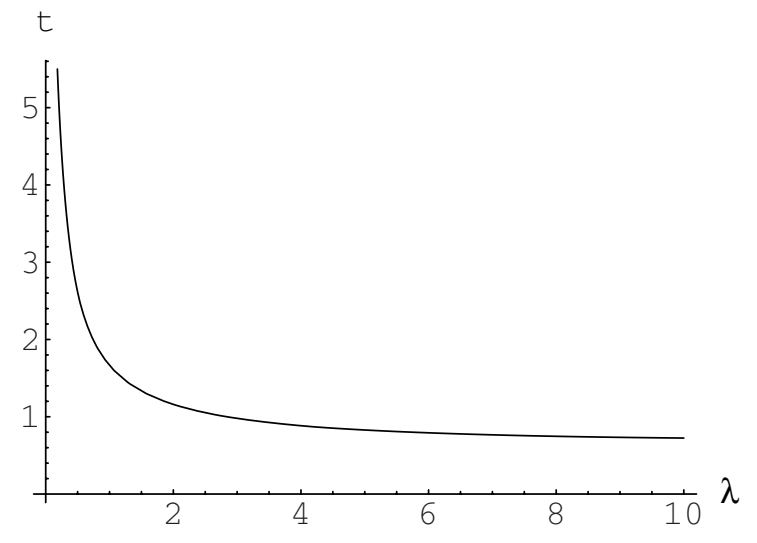

Fig. 2: Phase transition defined by $m\left(T_{c}\right)=0$ : values for the pair $\left(T_{c}, \lambda_{0}\right)$ define the critical line; on the vertical axis $t=$ $T_{c} / m_{0}$ and on the horizontal axis $\lambda=\lambda_{0} m_{0} / 2 \pi$. The minimum critical temperature is $T_{c} \simeq 0.874 m_{0}$.

that indeed there is a second-order phase transition, characterized by the divergence of the four-point function and with the critical temperature obtained from the condition $m\left(T_{c}\right)=0$.

A further indication of a second-order transition can be obtained from a renormalization group point of view, following lines analogous to those employed in [35] for type-II superconducting films. If an infrared stable fixed point exists, it is possible to determine it by a study of the infrared behaviour of the beta-function, i.e., in the neighbourhood of vanishing external momentum $|p|=0$; we consider the thermal coupling constant at criticality, with an external small momentum, given by

$$
g(T,|p| \approx 0)=\frac{\lambda_{0}}{\left[1-\lambda_{0} \Pi(D, T, p)\right]} .
$$

Using a Feynman parameter $x$, the one-loop four-point function is

$$
\begin{gathered}
\Pi(D, T, p)=\frac{1}{\beta} \sum_{n=-\infty}^{\infty} \int_{0}^{1} \mathrm{~d} x \int \frac{\mathrm{d}^{D-1} k}{(2 \pi)^{D-1}} \frac{M_{0}^{2}-\left(k^{2}+\omega_{n}^{2}\right)}{\left(k^{2}+\omega_{n}^{2}+M_{0}^{2}\right)^{2}} \\
-\frac{p^{2}}{\beta} \sum_{n=-\infty}^{\infty} \int_{0}^{1} \mathrm{~d} x \int \frac{\mathrm{d}^{D-1} k}{(2 \pi)^{D-1}} \frac{x(1-x)}{\left(k^{2}+\omega_{n}^{2}+M_{0}^{2}\right)^{2}},
\end{gathered}
$$

where

$$
M_{0}^{2}(p)=m_{0}^{2}+p^{2} x(1-x) ; \quad a_{c}(p)=\frac{1}{\beta^{2} M_{0}(p)} .
$$

The second term in eq. (30) vanishes in the limit $|p| \rightarrow 0$. Following the calculation steps for small values of $|p|$ detailed in [35], we are left for $|p| \approx 0$, after regularization, with an expression similar to eq. (22),

$$
\begin{aligned}
\Pi_{R}(D, T, p)= & \frac{M_{0}^{D-2}}{2^{D-2} \pi^{D / 2}}\left[2 \pi W\left(2, a_{c}(p), D\right)\right. \\
& \left.-W\left(1, a_{c}(p), D\right)\right]
\end{aligned}
$$


where it is understood that we take asymptotic values for small values of the argument in the Bessel functions in eq. (20), which defines the quantity $W(\mu, a, D)$.

For $D=3$ we have, $M_{0}^{D-2} \approx m_{0}+\frac{1}{2} p^{2} x(1-x)$, and

$$
\Pi_{R}(3, T, p)=B(3, T)+A(3, T)|p|,
$$

where

$$
B(3, T)=\frac{m_{0}}{2 \pi^{3 / 2}}\left[2 \pi W\left(2, a_{c}, 3\right)-W\left(1, a_{c}, 3\right)\right]
$$

and

$$
A(3, T)=\frac{a}{2 \pi^{3 / 2}}\left[2 \pi W\left(2, a_{c}, 3\right)-W\left(1, a_{c}, 3\right)\right]
$$

with $a=\int_{0}^{1} \mathrm{~d} x \sqrt{x(1-x)}=\pi / 8$.

The coupling constant has dimension of $|p|^{-1}$; taking $|p|$ as a running scale we define a dimensionless coupling constant

$$
g^{\prime}=|p| g=\frac{|p| \lambda_{0}}{1-\lambda_{0}[B(T, 3]+A(T, 3)|p|}
$$

and the beta-function,

$$
\beta\left(g^{\prime}\right)=|p| \frac{\partial g^{\prime}}{\partial|p|} ;
$$

we easily see that the condition of a non-trivial infrared stable fixed point is fullfiled by the solution

$$
g_{\star}^{\prime}=\frac{1}{A\left(3, T_{c}\right)} .
$$

As we have already stated in this note, the GN model may be seen as an effective theory for QCD, with an arbitrary zero-temperature coupling constant. Let us then estimate a specific value for the critical temperature, choosing the mass of the Gross-Neveu fermion to be the effective quark mass of the proton [36], $m_{0} \approx 330 \mathrm{MeV}$. Consider a strong coupling regime characterized by large values of $\lambda_{0}$, up to the limit $\lambda_{0} \rightarrow \infty$ (see fig. 2). For these values (for instance for $\lambda_{0} \approx 16 \pi / m_{0}=0.15 \mathrm{MeV}^{-1}$, corresponding to $\lambda=8$ in fig. 2), we find a critical temperature, $T_{c}$, of the order of $T_{c} \sim 0.87 m_{0} \approx 288 \mathrm{MeV}$.

Concluding remarks. - In short, we have answered positively the question whether there is a phase transition in the $N=1$ massive GN model; i.e. yes, there is a second-order phase transition. This conclusion is based in two results. From the non-perturbative analysis of the four-point function, a critical region can be established. Thereby the analysis of the free energy provides the nature of the transition, as being of second order. It is important to add that this procedure is strikingly similar in character to the method for finding critical temperature for the second-order phase transition in BCS model of superconductivity. Also, we have shown that we can define a beta-function, which has a non-trivial infrared fixed point, thus reinforcing the conclusion that the transition is a second-order one.

Considering a regime of strong coupling as defined above, and the mass of the GN fermion as the effective quark mass, we find a critical temperature of the order of $T_{c} \simeq 288 \mathrm{MeV}$. It is interesting to observe that this value is of the same order of magnitude as the estimated temperature $\sim 200 \mathrm{MeV}$ for the quark deconfinement transition, obtained from lattice calculations. The phase transition here may be then associated with the transition from a hadronic state to a quark-gluon plasma.

This has been possible by employing the massive GN model in three dimensions, which has been shown to exist and constructed for the first time in [19]. In previous works devoted to get insights on the behaviour of hadronic matter [2-13,15-17], the massless GN model, in its version with a large number of components, has been often employed. Using the one-component massive GN model in three dimensions and taking the fermion mass as a physical parameter (the effective quark mass), we have been able using analytical means, to determine the transition temperature. Moreover this temperature is found to fall into a range of values compatible with the transition temperature for hadronization, estimated from lattice simulations. A rigorous study involving additional aspects of this transition, including determination of critical exponents, is left for future work.

Finally, we would like to add some comments about the suitability of the GN model to fit QCD. We have in mind the Nambu-Jona-Lasinio (NJL) model which, particularly in its four-dimensional version, is closer to QCD than the GN model. Many studies on the phase structure of the two- and four-dimensional NJL model have been performed, which includes articles by some of us (see $[37,38]$ and references therein). A relevant aspect of the massive three-dimensional GN model, as we have employed in this article, is that the critical temperature can be obtained analytically in the context of a well-known phenomenological approach to phase transitions. This is much harder to be studied with the NJL model; it will be the subject of future work.

This work received partial financial support from CAPES, CNPq, FAPERJ (Brazil) and NSERC (Canada).

\section{REFERENCES}

[1] Gross D. J. and Neveu A., Phys. Rev. D, 10 (1974) 3235.

[2] Barducci A., Casalbuoni R., Modugno M., Pettini G. and Gatto R., Phys. Rev. D, 51 (1995) 3042.

[3] Christiansen H. R., Petkou A. C., Silvaneto M. B. and Vlachos N. D., Phys. Rev. D, 62 (2000) 025018.

[4] Hands S. J., Kogut J. B. and Strouthos C. G., Phys. Lett. B, 515 (2001) 407. 
[5] Hands S. J., Kogut J. B. and Strouthos C. G., Phys. Rev. D, 65 (2002) 114507.

[6] Thies M., J. Phys. A, 39 (2006) 12707.

[7] Schnetz O., Thies M. and Urlichs K., Ann. Phys. (N.Y.), 321 (2006) 2604.

[8] Juričić V., Herbut I. F. and Semenoff G. W., Phys. Rev. B, 321 (2009) 081405.

[9] Zhou B. R., Phys. Rev. D, 57 (1998) 3171.

[10] Zhou B. R., Commun. Theor. Phys., 32 (1999) 425.

[11] Brzoska A. and Thies M., Phys. Rev. D, 65 (2002) 125001.

[12] Kneur J.-L., Pinto M. B., Ramos R. O. and Staudt E., Phys. Rev. D, 76 (2007) 045020.

[13] Boehmer C., Fritsch U., Kraus S. and Thies M., Phys. Rev. D, 78 (2008) 065043.

[14] Höfling F., Nowak C. and Wetterich C., Phys. Rev. $B, 66$ (2002) 205111.

[15] Malbouisson A. P. C., Malbouisson J. M. C., Santana A. E. and Silva J. C., Phys. Lett. B, 583 (2004) 373.

[16] Khanna F. C., Malbouisson A. P. C., Malbouisson J. M. C., Queiroz H., Rocha-Filho T. M., Santana A. E. and Silva J. C., Phys. Lett. B, 624 (2005) 316.

[17] Konyama H., Phys. Rev. D, 77 (2008) 045016.

[18] Feinberg J., Int. J. Mod. Phys., 17 (2002) 898.

[19] de Calan C., Faria da Veiga P. A., Magnen J. and SÉneor R., Phys. Rev. Lett., 66 (1991) 3233.

[20] Gawedzki K. and Kupiainen A., Phys. Rev. Lett., 55 (1985) 363.

[21] Gawedzki K. and Kupiainen A., Nucl. Phys. B, 262 (1985) 33.

[22] Weinberg S., Phys. Rev. D, 56 (1997) 2303.
[23] Parisi G., Nucl. Phys. B, 100 (1975) 368.

[24] Arefŕa Y., Nissimov E. and Pacheva S., Commun. Math. Phys., 71 (1980) 213.

[25] Rosenstein B., Warr B. J. and Park S. H., Phys. Rev. Lett., 62 (1989) 1433.

[26] Doniach S. and Sondheimer E. H., Green's Functions for Solid State Physicists (W.A. Benjamin, London) 1974.

[27] Ramond P., Field Theory - a Modern Primer (AddisonWesley, Redwood) 1990.

[28] Kirzhnits D. A. and Linde A. D., Ann. Phys. (N.Y.), 101 (1976) 195.

[29] Khanna F. C., Malbouisson A. P. C., Malbouisson J. M. C. and SAntanA A. E., Thermal Quantum Field Theory: Algebraic Aspects and Applications (World Scientific, Singapore) 2009.

[30] Epstein P., Math. Ann., 56 (1902) 615.

[31] Elizalde E. and Romeo A., J. Math. Phys., 30 (1989) 1133.

[32] Malbouisson A. P. C. and Malbouisson J. M. C., J. Phys. A: Math. Gen., 35 (2002) 2263.

[33] Malbouisson A. P. C., Malbouisson J. M. C. and Santana A. E., Nucl. Phys. B, 631 (2002) 83.

[34] Malbouisson A. P. C., Khanna F. C., Malbouisson J. M. C. and Santana A. E., Braz. J. Phys., 36 (2006) 1165.

[35] Malbouisson A. P. C., Phys. Rev. B, 66 (2002) 092502.

[36] Maltman K. and Isgur N., Phys. Rev. D, 29 (1984) 952.

[37] Abreu L. M., Malbouisson A. P. C., Malbouisson J. M. C. and Santana A. E., Nucl. Phys. B, 819 (2009) 127.

[38] Abreu L. M., Malbouisson A. P. C. and Malbouisson J. M. C., EPL, 90 (2010) 11001. 